\title{
POLYGLOT: A MULTILINGUAL GROUP SUPPORT SYSTEM
}

\author{
Milam Aiken, University of Mississippi, aiken@ bus.olemiss.edu \\ Mahesh Vanjani, Texas Southern University, vanjanim@tsu.edu
}

\begin{abstract}
This paper describes a new, locally developed Internet-based electronic group support system that automatically translates among 34 languages through a linkage with Google Translate. The overall accuracy rate was only $86 \%$, but near perfect accuracy might not be needed for informal, information gathering group meetings in which ideas are often redundant and can be rephrased. Further, the translation speed advantage might offset this weakness.
\end{abstract}

Keywords: Polyglot, Multilingual, Group Support System, GSS, Machine Translation, MT

\section{INTRODUCTION}

Group support systems (otherwise known as group decision support systems or electronic meeting systems) enhance information-sharing meetings by providing parallel input of anonymous ideas that are recorded to a file [4], and many studies of meetings using English for communication have shown that these discussions are often more efficient and effective when using this computer-based technology [7].

However, many meetings around the world (e.g., conferences within the United Nations) involve participants speaking many different languages. In a global economy, three out of four multinational companies now manage networks of 20 or more overseas operations with employees, customers, and suppliers speaking a large number of languages [9]. Groups in which participants do not speak the same language frequently find communication difficult. Yet, multilingual meetings are common as a form of collaboration. To overcome this language barrier, banks, government agencies, hospitals, the courts, and many other institutions have relied upon human translators to enable meeting participants to exchange ideas and opinions. For example, the United Nations General Assembly's discussions, conferences within the European Union, multinational corporations' business negotiations, and many other meetings are conducted almost daily, requiring large amounts of interpreters' scarce expertise and time.
In addition to the problems of translation efficiency and effectiveness, these meetings have the same limitations as those involving a single language, e.g., (1) only one participant can speak at a time, (2) comments must be transcribed manually for a permanent record, and (3) many group members do not participate because of shyness or because other speakers monopolize the conversation.

Group Support Systems (GSS) have automated the meeting process and improved the productivity of groups needing to share ideas [4]. Integrating machine translation (MT) with group support systems can provide support for multilingual meetings. If machine translation (MT) is integrated with group support systems, these multilingual groups could be more productive as well by eliminating the need for participants to take turns speaking, enhancing participation, and improving the overall satisfaction with the discussions.

Research in MT began soon after World War II, but the first proposal to use it in conjunction with an electronic meeting was made about 20 years ago [8]. The first fully automated multilingual group support system translating between Spanish and English appeared a few years later [2], and Web-based versions of the group meeting technology began to appear a few years after that $[5,13]$. In these fully automatic Multilingual Group Support System (MGSS) meetings, participants were able to type in one language and submit the comment while translations automatically appeared on other terminals. Based upon software configuration, group members could be allowed to view comments only in their language (e.g., Spanish) or comments in a mixture (e.g., Spanish and English). In the latter approach, if a translation was inaccurate and a participant knew a little of the other language, he or she could possibly make a more accurate guess as to the correct meaning of the comment.

Several versions of MGSSs appeared subsequently with support for more languages and increasing accuracy [1, 14]. Machine translation is the basis of an MGSS meeting, and its comprehension potential is vital to the success of the discussion. This paper describes the newest version of a multilingual group support system (MGSS) capable of translating among 
34 languages with high understandability, even if many passages are ungrammatical and awkward.

\section{BACKGROUND AND GOOGLE TRANSLATE}

\section{MT Programs}

Providing support for multilingual, verbal meetings is expensive and difficult. A linguist can charge \$US .10 - .15 per word [6] or $\$ 20$ per hour [10] for each language that is supported, and assuming a one-hour minimum charge, an interpreter for each of 10 meeting participants could cost \$200. Further, and much more challenging, arranging for multiple interpreters (especially for obscure languages) to appear at one time in a remote location can be extremely difficult.

Although not as accurate as human interpreters, several commercially available translation programs have been developed, and several free versions have even appeared on the Web, e.g.:

- Applied Language

(http://www.appliedlanguage.com/free_translatio n.shtml)

- SDL Automated Translation Solutions (http://www.freetranslation.com/)

- Windows Live Translator (http://translator.live.com)

- Yahoo! Babel Fish (http://babelfish.yahoo.com)

With the advent of Google Translate (http://translate.google.com/translate_t\#), accuracies have increased substantially. Abandoning a grammarrule approach, the service uses statistical learning techniques to build a language model from an analysis of billions of words of parallel text. That is, text in a German Bible can be compared with text in a Korean Bible to extract statistical probabilities of words and phrases in one language ending up as particular words or phrases in another. New text is then evaluated using this language-pair model.

Google Translate's results have been extremely encouraging. In the most recent National Institute of Standards and Technology (NIST) test of 22 MT systems [11], the system was often first and never lower than third in the rankings based upon BLEU [12] scores of text translated from Arabic to English and from Chinese to English.

The NIST 2006 Machine Translation Evaluation (MT-06) was part of an ongoing series of evaluations of human language translation technology. NIST conducts these evaluations in order to support machine translation (MT) research and help advance the state-of-the-art in machine translation technology. These evaluations provide an important contribution to the direction of research efforts and the calibration of technical capabilities. The MT-06 evaluation consisted of two tasks. Each task required a system to perform translation from a given source language into the target language. The source languages were Arabic and Chinese, and the target language was English [11].

However, few if any tests have been conducted on all the language pairs. Although it might be possible to arrange Spanish, German, or even Japanese speakers to evaluate translations in these languages, it is extremely difficult for us to find Latvian, Bulgarian, or Vietnamese speakers. In an attempt to get a better measure of the potential accuracy for all 33 nonEnglish languages provided with this MT system within an electronic meeting, we devised a reversetranslation test.

In mathematics, the symmetric property states that for any quantities $a$ and $b$, if $a=b$, then $b=a$, and the transitive property states that for any quantities $a, b$, and $c$, if $a=b$ and $b=c$, then $a=c$. Used in the realm of language translation, the symmetric property states that if a translation of text $a$ in language $X$ gives text $\mathrm{b}$ in a language $Y$, then a translation of text $b$ to language $\mathrm{X}$ should give text $a$. Likewise, using the transitive property, text should be equivalent when translated into three different languages in a circular manner.

If a translation program or service exhibits symmetric and transitive properties, it is easy to verify the accuracy, even if the evaluator knows only the source language, e.g. English. For example, if a program translates "Good morning" to "Guten Morgen," and translates "Guten Morgen" to "Good Morning," an English speaker does not need to know German to believe the program is reasonably accurate, for this particular phrase. Of course, a much more comprehensive sample of phrases in a variety of topics must be tested before final judgment. Further, this accuracy should be a lower bound, as each translation between language pairs can provide additional errors.

\section{Evaluation}

Ten edited sentences from an electronic meeting transcript of college students discussing solutions for the parking problem on campus were used for the evaluation:

1. Build a parking garage for student parking. 
2. The parking at the union needs to be the way that it was with 30-minute limits.

3. I think that the parking should be next to the Honors College.

4. The law school should be open to those who do not live on campus.

5. Get a shuttle bus that assists students to class.

6. A parking garage would be great.

7. A parking garage is probably the best way to improve parking.

8. There is too much faculty and staff parking.

9. Don't sell more decals than there are spaces.

10. Divide the parking lots into categories of age and majors.

These phrases were translated into each non-English language and that translation was converted back into English using Google Translate. For example, the previous 10 sentences converted into Korean resulted in:

1. 학생 주차장에 대한 주차장을 빌드합니다.

2. 노조에서 30 - 분 주차 그 한계가 같이 있던 방법이있을 필요가있다.

3. 나는 명예를 대학 주차장 옆에 있어야한다고 생각합니다.

4. 로스쿨 캠퍼스에 살지 않아 자들에게 열려 있어야합니다.

5. 그 수업에 학생을 돕습 셔틀 버스를 타고있다.

6. 주차장 대단한 것이다.

7. 아마 주차장 주차장을 개선하는 최선의

방법입니다.

8. 이미 너무 많은 교수진과 직원 주차장입니다.

9. 거기에 공백보다 더 데칼 판매하지 마십시오.

\section{0. 나이와 전공의 범주에 주차장으로}

나누십시오.

A native Korean speaker stated that sentences 1, 6, 7, 8,9 , and 10 were very clear, and 2, 4, and 5 had errors but were understandable. Sentence 3 was not understandable as a whole, but individual words and fragments were clear.

These Korean sentences converted back to English resulted in:

1. To build a parking lot in the student parking lot.

2. The union in the 30 - minute parking was with the limitations it is necessary to be a way.
3. I think I have the honor to the university next to the parking lot.

4. Law must be open to those who do not live on campus.

5. The shuttle bus ride to class, students said.

6. Parking will be great.

7. Perhaps the best way to improve the parking lot is a parking lot.

8. Faculty and staff parking lot is already too much.

9. Do not sell more than a space in there decals.

10. Major in the category of age and over to the parking lot to share.

Phrases 3 and 10 were judged to be not understandable. Thus, two phrases were not understood in the round-trip (translation from English to Korean followed by translation from Korean to English), but only one phrase was not understood in the conversion to Korean. Of course, the sample was limited, and individual evaluators have different subjective opinions about what is understandable.

Likewise, each of the final English reverse translations from the 33 foreign languages was evaluated by a native English speaker, and the results are shown in Table 1.

Table 1: Google Translate

Foreign Languages Supported and Accuracies (10 sentences translated from English to the language, and back to English)

\begin{tabular}{|c|c|c|c|}
\hline 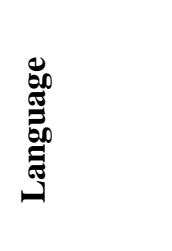 & 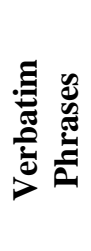 & 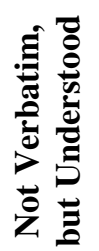 & 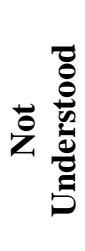 \\
\hline Arabic & 1 & 9 & 0 \\
\hline Bulgarian & 0 & 9 & 1 \\
\hline Catalan & 2 & 6 & 2 \\
\hline Chinese & 0 & 7 & 3 \\
\hline Croatian & 2 & 8 & 0 \\
\hline Czech & 3 & 5 & 2 \\
\hline Danish & 4 & 5 & 1 \\
\hline Dutch & 3 & 7 & 0 \\
\hline Filipino & 0 & 9 & 1 \\
\hline Finnish & 3 & 6 & 1 \\
\hline French & 1 & 7 & 2 \\
\hline German & 1 & 7 & 2 \\
\hline Greek & 2 & 7 & 1 \\
\hline Hebrew & 0 & 9 & 1 \\
\hline Hindi & 0 & 6 & 4 \\
\hline Indonesian & 0 & 9 & 1 \\
\hline
\end{tabular}




\begin{tabular}{|l|c|c|c|}
\hline Italian & 3 & 5 & 2 \\
\hline Japanese & 0 & 5 & 5 \\
\hline Korean & 0 & 8 & 2 \\
\hline Latvian & 2 & 7 & 1 \\
\hline Lithuanian & 1 & 7 & 2 \\
\hline Norwegian & 2 & 8 & 0 \\
\hline Polish & 0 & 10 & 0 \\
\hline Portuguese & 0 & 10 & 0 \\
\hline Romanian & 3 & 6 & 1 \\
\hline Russian & 0 & 8 & 2 \\
\hline Serbian & 0 & 8 & 2 \\
\hline Slovak & 0 & 9 & 1 \\
\hline Slovenian & 2 & 7 & 1 \\
\hline Spanish & 2 & 7 & 1 \\
\hline Swedish & 4 & 5 & 1 \\
\hline Ukrainian & 0 & 9 & 1 \\
\hline Vietnamese & 2 & 6 & 2 \\
\hline \multicolumn{1}{|c|}{ Total } & $\mathbf{4 3}$ & $\mathbf{2 4 1}$ & $\mathbf{4 6}$ \\
\hline Average & $\mathbf{1 . 3}$ & $\mathbf{7 . 3}$ & $\mathbf{1 . 4}$ \\
\hline
\end{tabular}

This resulted in 330 reverse translated comments. Overall, $13 \%$ of these reverse translations were verbatim, $73 \%$ of these reverse translations were not verbatim but understood; and, $14 \%$ of these reverse translations were judged as not understood.

Many of the $73 \%$ "understood" comments were very similar, e.g.:

- Italian: "A parking lot is probably the best way to improve parking." instead of "A parking garage may be the best way to improve parking."

- Latvian: "I think that parking should be next to the Honors College." instead of "I think that the parking should be next to the Honors College."

- Lithuanian: "A parking garage will be great." instead of "A parking garage would be great."

- Polish: "A garage would be great." instead of "A parking garage would be great."

Roughly half of the understood comments were worded strangely or grammatically incorrect, but still understood, e.g.:

- Hindi: "Parking for students to create a parking garage."

- Indonesian: "I think, should be parked on the side Honors College."

- Japanese: "Too many of the faculty and staff parking."

- Korean: "Law must be open to those who do not live on campus."

Examples of the $14 \%$ misunderstood comments include:
- Catalan: "Parking is probably the best way to improve parking."

- Filipino: "The parking needs of the union that this method has a 30-minute limit."

- Finnish: "The parking area at European Union level must be taken so that it was a 30 -minute limits."

- French: "Do not sell more than places thumbnails."

- German: "A parking garage would großartig."

- Greek: "Sell no more than decals gaps."

- Serbian: "Get the bus station, which helps students in the class."

- Vietnamese: "Chia parking lots in the categories of age and majors."

Interestingly, "union" (i.e., student union) was interpreted in several cases by some European languages to be "European Union." Only two instances of unknown words appeared (the German word "großartig" - great or wonderful, and the Vietnamese word "chia" - split up into small pieces), and it is somewhat surprising that these relatively common words were not in the system dictionaries.

Some reverse translations made sense, but conveyed the wrong meaning, and these were counted as misunderstood, e.g. Indonesian: "A parking garage will be large." instead of "A parking garage would be great."

The worst results were from the Japanese and Hindi translations, perhaps because of the characteristics of these languages or inadequate language-pair models. On the other hand, some of the best results came from European languages very similar to English, including Dutch, Danish, and Swedish which had three to four verbatim reverse translations. A few other very dissimilar languages such as Arabic and Polish also provided good results.

\section{POLYGLOT}

\section{System Description}

Polyglot ("many tongues") was developed with a Microsoft Windows client-server architecture incorporating UDP (User Datagram Protocol), FTP (File Transfer Protocol), and HTTP (Hyper Text Transport Protocol) linkages between the components (see Figure 1). To use the system, meeting participants

1. Run the client program and type in the IP address of the server, 
2. Select "Connect" in the menu, connecting the client application to the server,

3. Type their assigned user numbers in a popup input box,

4. Select a language (e.g. Korean) in the menu drop-down box,

5. Type a comment in the language and press the INSERT key, which automatically translates the text to English by querying Google Translate, sends it to the server, and adds the comment in English to the transcript in a Web server file and a file on the server recording the user number, time submitted, and the text,

6. Read meeting comments contributed thus far which are converted via Google Translate from English back to the language the participants selected in step \#4, and

7. Continue submitting new comments until the meeting is over.

Figure 1: The Multilingual GSS Meeting Process

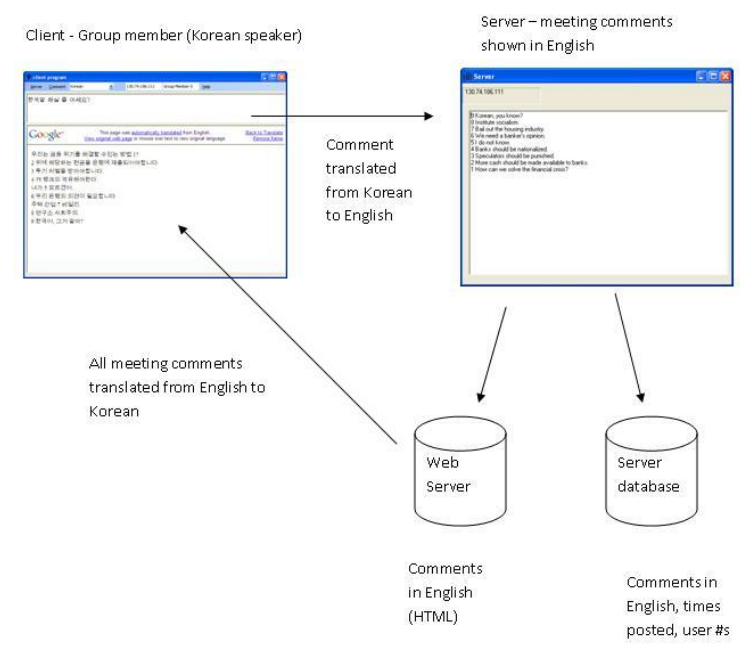

Because translation queries to Google Translate are from multiple client programs, the server application is not overwhelmed by several simultaneous translation requests. Individual comments take one or two seconds to translate into English, and a complete meeting transcript translation back into the client's selected language (after each new comment submission) takes a maximum of 10 seconds. Participants can still type new comments even while waiting for the meeting transcript to refresh on their screens.

The transcript appears in English in a textbox on the server program, and the meeting facilitator can add comments such as "Please finish the meeting now." This will appear on the users' client programs in their native languages after they contribute their next comment. In addition, the facilitator has the option of editing the contributed comments in English within the server program, enhancing the translation accuracy.

\section{Discussion}

Although the understanding accuracy of the 10 reverse-translated comments averaged $86 \%$, several languages provided $90 \%$ or $100 \%$. In addition, the meaning of individual words and fragments often can be ascertained even if the overall sentence is misunderstood. For example, the misunderstood translation from Japanese "Perhaps in the parking lot to improve the parking lot is the best way." states that "a parking lot is the best way." Extra hints as to the meaning can be provided by other, perhaps redundant comments. Finally, within an informal, informationgathering electronic meeting, it probably is not essential that every comment is translated accurately. That is, there is no penalty for information loss, especially since the alternative is no translation at all.

In an actual meeting, however, participants are likely to make some spelling and grammatical errors, reducing the accuracy. In addition, the use of idioms, slang, and jargon can reduce the understandability. Future research should investigate how much the use of these terms reduces the understandability of comments.

Accuracy is only one consideration in an MGSS meeting, however; translation speed is also important. For example, the value of a translation might be expressed as a product:

Value $=$ speed $*($ importance of speed $) *$ accuracy $*$ (importance of accuracy)

Humans can translate at about 650 words per hour or .18 words per second [3], not including the time to type the text, while Google Translate's speed appears to be about 155 words per second.

Table 2: Value of Translations

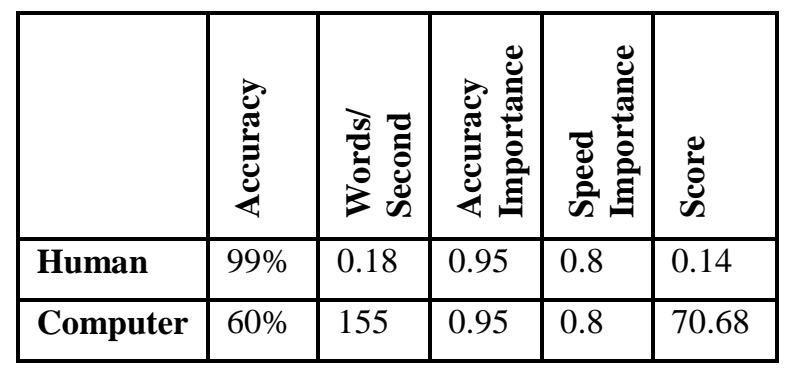


As the hypothetical case in Table 2 shows, even if an MT system's translation accuracy is only $60 \%$ while a human's is $99 \%$, if the importance of speed is 0.8 on a scale of 0 to 1 and the importance of accuracy is 0.95, then MT might be superior. In addition, Google Translate's accuracy continues to improve as more text is provided for statistical analysis.

Finally, future versions of the program might include direct translations between language pairs (e.g., German to Korean or Chinese to Danish) instead of each comment first being converted into English. Having a single comment file in English reduces the complexity, and only one translation needs to be done after each comment is written, regardless of the number of languages in the meeting. However, each time a language is translated (e.g. Chinese to English and then English to German) some additional error can be added.

\section{CONCLUSION}

Lingual barriers to communication in group meetings reduce their efficiency and effectiveness, but a new multilingual group support system can lower these barriers through fast and relatively understandable translations. Future research will focus on use of the MGSS in real-life meetings featuring participants typing several different languages at different locations.

\section{REFERENCES}

1. Aiken, M. (2008). Multilingual Collaboration in Electronic Meetings. in Encyclopedia of ECollaboration, Information Science Reference: Hershey, PA

2. Aiken, M., Martin, J., Reithel, B., Shirani, A., and Singleton, T. (1992). Using a Group Decision Support System for Multicultural and Multilingual Communication. Proceedings of the 23rd Annual Meeting of the Decision Sciences Institute, November, San Francisco, CA, 792794.

3. Bass, S. (1999). Machine vs. Human Translation. Advanced Language Translation, Inc., Available from: $\quad$ http://www.advancedlanguage.com [accessed 10/28/08]

4. Dennis, A., George, J., Jessup, L., Nunamaker, J., and Vogel, D. (1988). Information Technology to Support Electronic Meetings. MIS Quarterly, 12(4), 591-624.

5. Dennis, A., Pootheri, S., and Natarajan, V. (1998). Lessons from the Early Adopters of Web-Groupware. Journal of Management Information Systems, 14(4), 65-86.

6. Embregts, D. (2008). Daniel Embregts: Professional Engineer and Translator. Available from: http://hezee.com/prices.html [accessed 10/28/08]

7. Fjermestad, J. and Hiltz, S. (2000). Group support systems: A Descriptive Evaluation of Case and Field Studies. Journal of Management Information Systems, 17(3), 115-159.

8. Gray, P. and Olfman, L. (1989). The User Interface in Group Decision Support Systems. Decision Support Systems, 5, 119-137.

9. John, R., Ietto-Gillies, G., Cox, H., and Grimwade, N. (1997). Global Business Strategy. International Thomson Press, London.

10. Language123 (2008). The Translation Marketplace: Language123. Available from: http://language123.com/l/translation_salary.html accessed 10/28/08]

11. NIST (2006). National Institute of Standards and Technology: Machine Translation Evaluation Official Results. Available from: http://www.nist.gov/speech/tests/mt/2006/doc/mt 06eval_official_results.html [accessed 10/28/08]

12. Papineni, K., Roukos, S., Ward, T., and Zhu, W. (2002). BLEU: A Method for Automatic Evaluation of Machine Translation. Proceedings of the $40^{\text {th }}$ Annual Meeting of the Association for Computational Linguistics, July, Philadelphia, PA, 311-318.

13. Romano, N., Nunamaker, J., Briggs, R., and Vogel, D. (1998). Architecture, Design, and Development of an HTML/JavaScript WebBased Group Support System. Journal of the American Society for Information Science, 49(7), 649-667.

14. Yamashita, N. and Ishida, T. (2006). Effects of Machine Translation on Collaborative Work. Proceedings of the 2006 20th Anniversary Conference on Computer Supported Cooperative Work, Banff, Alberta, Canada, 515 - 524. 